Case Report

\title{
Acupuncture for Management of Symptom Clusters in a Patient with Metastatic Breast Cancer: A Case Report
}

Tina Huang ${ }^{1,+}$, Tony Hung ${ }^{2,+, *}$, S. Irene Kim ${ }^{2,+}$, Ka Kit Hui ${ }^{2,+}$

1. David Geffen School of Medicine at UCLA, 10833 Le Conte Ave, Los Angeles, CA 90095, U.S.A.; EMail: TQHuang@mednet.ucla.edu

2. UCLA Center for East-West Medicine, 1015 Gayley Ave, Suite 301, Campus Mail Code: 735607 Los Angeles, CA 90024, U.S.A.; E-Mails: tonyhung.ucla@gmail.com; s.irenekim@gmail.com; KHui@mednet.ucla.edu

† These authors contributed equally to this work.

* Correspondence: Tony Hung; E-Mail: tonyhung.ucla@gmail.com

Academic Editor: Im Quah-Smith

Special Issue: Acupuncture for Women's and Children's Health

OBM Integrative and Complementary Medicine

2020 , volume 5 , issue 1

doi:10.21926/obm.icm.2001011
Received: October 07, 2019

Accepted: February 25, 2020

Published: March 02, 2020

\begin{abstract}
Acupuncture has increasingly been used to address a variety of symptoms in patients with cancer. Here we present a case to illustrate the use of acupuncture in a 35-year-old female with metastatic breast cancer who presented to the UCLA Center for East-West Medicine (CEWM) with physical and psychological symptom clusters of insomnia, anxiety, and joint pain.
\end{abstract}

\section{Keywords}

Acupuncture; integrative east-west medicine; breast cancer; case report

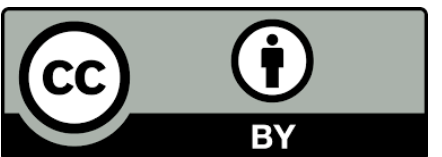

(C) 2020 by the author. This is an open access article distributed under the conditions of the Creative Commons by Attribution License, which permits unrestricted use, distribution, and reproduction in any medium or format, provided the original work is correctly cited. 


\section{Introduction}

Acupuncture is a traditional Chinese medicine practice that involves the manual stimulation of thin, solid, metallic needles inserted into acupuncture points. Over the recent decades, acupuncture has been recognized and increasingly been used as a non-pharmacological intervention for variety of cancer-associated symptoms in the United States. [1-5] Here we present a case to illustrate the use of acupuncture in a 35-year-old female with metastatic breast cancer who presented to the UCLA Center for East-West Medicine (CEWM) with physical and psychological symptom clusters of insomnia, anxiety, and joint pain.

\section{Case}

A 35-year-old female with a history of Stage IIB (T2N1M0) hormone receptor-positive (ER+, $\mathrm{PR}+$ ), HER2-negative (HER2-) invasive ductal carcinoma of the right breast with bone and lung recurrence presented to CEWM with a twelve months history of worsening joint pain, insomnia, and anxiety. She was first diagnosed in 2013 (BRCA mutation testing was negative) and underwent right breast lumpectomy, followed by adjuvant chemotherapy. Radiation was offered but the patient declined treatment. Adjuvant tamoxifen was started thereafter, but she was not able to tolerate treatment due to its side effects and ended treatment after a brief exposure. Other than a history of oral contraceptive exposure since age 14, she has no other relevant medical history. She is of Ashkenazi Jewish descent, and her family history is significant for two paternal aunts with breast cancer.

Patient was followed expectantly. In 2017, she reported a nodule $8 \mathrm{~mm}$ in size at the 3 o'clock position of her right breast. Biopsy of this nodule confirmed local recurrence of breast cancer (ER+/PR+, HER-). Her exam and laboratory were otherwise non-contributory. Staging imaging found an additional $5 \mathrm{~mm}$ left lower lung nodule and a $6 \mathrm{~mm}$ sclerotic lesion of the sternum with adjacent cortical erosion, both suspicious for metastases.

Following, she was started on cancer-directed treatment including Leuprolide, Letrozole, Palbociclib, and Denosumab. Several months into therapies, she began to develop notable symptoms including insomnia, anxiety, and pain of her joints, neck, and shoulders. Her joint pain was primarily in both knees, and described as "aching" and "stiff". Her anxiety increased with stress over her family life and her financial need to go back to work. Coupled with her frequent insomnia, her symptoms had been debilitating. As a result, she was referred to CEWM for further symptomatic management.

After an assessment by the team of East-West trained physicians, etiologies of her symptoms were thought to be due to both her cancer and treatment-related side effects. While nonspecific at first glance, her symptoms represented physical and psychological symptom clusters commonly seen in patients with breast cancer. In modern Western medicine, these symptom clusters may be attributed to hormonal imbalance, inflammatory and immune disturbance. Complementarily, drawing from principles of Traditional Chinese Medicine (TCM), her clinical manifestations suggest the TCM diagnoses of Spleen Qi deficiency and Liver Qi stagnation. Spleen Qi Deficiency in TCM contributed to patient's symptoms of chronic fatigue and insomnia, while Liver Qi stagnation contributed to her emotional irritability and anxiety. TCM theory dictates that "Toxic-heat" agents such as chemotherapy consumed and disrupted the smooth flow of the body's Qi. These effects 
subsequently led to a deficiency of Qi and blood, in addition to disharmony or impairment of his visceral function.

Based on the integrative assessment, the patient was offered acupuncture to manage her symptoms. Acupuncture points were selected to address the patient's specific symptoms and to rebalance the disharmony of her Qi based on TCM theory. Specifically, the acupuncture points used included LI-11, Du-20, Sp-9, Sp-10 LI-4/Lv-3, Sp-6/St-36, and P-6/Sp-4.

Treatment was scheduled with appointments every three to four weeks. Depending on the patient's overall progress, acupuncture points were personalized at each visit. Ongoing acupuncture, along with mindfulness meditation, was added in the latter course of her treatment and continued to alleviate her symptoms. Joint pain was first to improve, with a slow progressive improvement over the next three weeks. Her insomnia also gradually improved over the next few weeks and was ultimately resolved at fourteen months of post-treatment initiation. Anxiety proved to be the most resistant to treatment for our patient, likely due to additional psychosocioeconomic factors. Anxiety was resolved after a year of acupuncture. Post-treatment, the patient no longer endorsed any symptoms and has returned to work full time.

\section{Discussion}

Joint pain, insomnia, and anxiety are symptoms commonly reported by patients with breast cancer. Recent strategies for identifying and managing symptoms have employed categorization of symptoms into specific groups, called symptom clusters. [1] Originally defined by Dodd et al. as groups of three or more related symptoms, symptom clusters are now defined as two or more symptoms that relate to each other more than to others outside the cluster. [1] Categorizing symptoms by symptom cluster allows clinicians to more effectively target symptom treatment and even predict possible future symptoms. Furthermore, symptom clusters may influence treatment, quality of life, and even prognosis. [2] In breast cancer, one common symptom cluster is the "fatigue, pain, and disturbed sleep" cluster. [3] Different interventions for treating cancer symptom clusters include exercise-based multimodal interventions, analgesic imagery, cognitivebehavioral therapy, and acupressure and acupuncture-and are still being developed. $[4,5]$ In this case report, we report how one patient's symptoms of fatigue, joint pain, and insomnia symptoms within this common symptom cluster-are treated and alleviated by acupuncture.

Among breast cancer patients, arthralgia is associated with nearly $50 \%$ of patients on aromatase inhibitor therapy. [6] These arthralgias contribute to aromatase inhibitor therapy nonadherence, which is in turn associated with decreased disease-free survival. $[7,8]$ In a multicenter randomized clinical trial with 226 women, acupuncture produced statistically significant improvements in joint pain between baseline and six weeks compared to sham acupuncture and control. Acupuncture treatments used in this study were standardized based on the symptomatic joint location. [9] Non-arthralgia pain treatment through acupuncture received a recommendation grade of $\mathrm{C}$, suggesting that acupuncture can be considered as therapy for pain by the Society for Integrative Oncology (SIO) whose recommendations have been supported by the American Society of Clinical Oncology (ASCO). [10]

Acupuncture is also effective for cancer-related insomnia. Insomnia is a common side effect of breast cancer treatment. Its many psychological (negative mood, difficulty concentrating, memory problems) and medical consequences (irritable bowel syndrome, fibromyalgia, asthma) can 
appreciably decrease overall quality of life. [11, 12] Patients with breast cancer are particularly vulnerable to developing insomnia, as both female sex and advanced age are risk factors for insomnia. [11] While pharmacological treatments are available for insomnia, several studies have shown that cognitive behavioral therapies (CBT) and acupuncture may be as or more effective than pharmacological therapies in treating insomnia. [13] A recently published multicenter PCORI trial comparing acupuncture and CBT has shown promising results for acupuncture used in treating insomnia. [13] In this study, patients received one 30 minute acupuncture session with standardized acupoints: Bilateral: Shenmen (H.T.7), Sanyinjiao (Sp. 6); Midline: Baihui (Gv. 20), Shenting (Gv. 24); Unilateral: Shenmen (Auricular), Sympathetic (Auricular). [13] While CBT was more effective than acupuncture in treating insomnia, both acupuncture and CBT produced clinically meaningful reductions in the severity of insomnia. Acupuncture-mediated insomnia treatment lasted just as long as CBT-mediated treatment.

Of the many psychological consequences of cancer treatment, anxiety is particularly prevalent for patients with breast cancer. Up to $32 \%$ of patients with breast cancer experience anxiety, with rural patients at greater risk. [14] Anxiety lingers in patients years after the initial breast cancer diagnosis and is associated with a higher level of unmet need in survivors. [15] Addressing anxiety in both patients and survivors of breast cancer is important for maintaining health and wellness across the survivorship spectrum. [15] While the SIO most strongly recommends meditation for treating breast cancer-related anxiety, their guidelines also recommend considering acupuncture, albeit as a lower-graded therapy.

Acupuncture has also been studied for its efficacy in relieving many other cancer-related symptoms and side effects. Chief among these side effects is cancer-related pain. While trials studying acupuncture-related alleviation of cancer-related pain have not been conclusive, a recent systemic review of randomized clinical trials found that the use of acupuncture was significantly associated with a reduction in cancer-related pain, and decreased analgesic use. [16] Additionally, acupuncture has shown promise in improving symptoms including fatigue, indigestion, hot flashes, chemotherapy-induced nausea and vomiting, radiation-related xerostomia, and other cancer- or treatment-related ailments. [17-20]

\section{Conclusion}

Acupuncture has been used to address a variety of complex symptoms in patients with cancer. Our case illustrated that alongside with conventional medicine, acupuncture offers an effective and safe complementary treatment option to help alleviate symptoms for patients with breast cancer.

\section{Acknowledgments}

We thank and dedicate our manuscript to our patients, families, friends, and supporters of the UCLA Center for East-West Medicine.

\section{Author Contributions}

All authors contributed to the design, writing, and editing of the manuscript. 


\section{Funding}

UCLA Center for East-West Medicine

\section{Competing Interests}

The authors have declared that no competing interests exist.

\section{References}

1. Dodd MJ, Miaskowski C, Paul SM. Symptom clusters and their effect on the functional status of patients with cancer. Oncol Nurs Forum. 2001; 28: 465-470. http://www.ncbi.nlm.nih.gov/pubmed/11338755.

2. Kirkova J, Walsh D. Cancer symptom clusters - a dynamic construct. Support Care Cancer. 2007; 15: 1011-1013. doi:10.1007/s00520-007-0259-2

3. Byar KL, Berger AM, Bakken SL, Cetak MA. Impact of adjuvant breast cancer chemotherapy on fatigue, other symptoms, and quality of life. Oncol Nurs Forum. 2006; 33: E18-E26. doi:10.1188/06.ONF.E18-E26

4. Kwekkeboom KL. Cancer symptom cluster management. Semin Oncol Nurs. 2016; 32: 373-382. doi:10.1016/j.soncn.2016.08.004

5. Xiao C. The state of science in the study of cancer symptom clusters. Eur J Oncol Nurs. 2010; 14: 417-434. doi:10.1016/j.ejon.2010.05.011

6. Crew KD, Greenlee H, Capodice J, et al. Prevalence of joint symptoms in postmenopausal women taking aromatase inhibitors for early-stage breast cancer. J Clin Oncol. 2007; 25: 38773883. doi:10.1200/JCO.2007.10.7573

7. Chirgwin JH, Giobbie-Hurder A, Coates AS, et al. Treatment adherence and its impact on disease-free survival in the breast international group 1-98 trial of Tamoxifen and Letrozole, alone and in sequence. J Clin Oncol. 2016; 34: 2452-2459. doi:10.1200/JCO.2015.63.8619

8. Henry NL, Azzouz F, Desta Z, et al. Predictors of aromatase inhibitor discontinuation as a result of treatment-emergent symptoms in early-stage breast cancer. J Clin Oncol. 2012; 30: 936-942. doi:10.1200/JCO.2011.38.0261

9. Greenlee H, Crew KD, Capodice J, et al. Methods to standardize a multicenter acupuncture trial protocol to reduce aromatase inhibitor-related joint symptoms in breast cancer patients. J Acupunct Meridian Stud. 2015; 8: 152-158. doi:10.1016/J.JAMS.2015.03.006

10. Greenlee H, DuPont-Reyes MJ, Balneaves LG, et al. Clinical practice guidelines on the evidence-based use of integrative therapies during and after breast cancer treatment. CA Cancer J Clin. 2017; 67: 194-232. doi:10.3322/caac.21397

11. Fiorentino L, Ancoli-Israel S. Insomnia and its treatment in women with breast cancer. Sleep Med Rev. 2006; 10: 419-429. doi:10.1016/j.smrv.2006.03.005

12. Desai K, Mao JJ, Su I, et al. Prevalence and risk factors for insomnia among breast cancer patients on aromatase inhibitors. Support Care Cancer. 2013; 21: 43-51. doi:10.1007/s00520012-1490-z

13. Garland SN, Xie SX, DuHamel K, et al. Acupuncture versus cognitive behavioral therapy for insomnia in cancer survivors: A randomized clinical trial. JNCI J Natl Cancer Inst. 2019; 111: 1323-1331. doi:10.1093/jnci/djz050 
14. Dph S. Review: Anxiety in cancer patients. 2000; 83: 1261-1267. doi:10.1054/bjoc.2000.1405

15. Hodgkinson K, Butow P, Hunt GE, Pendlebury S, Hobbs KM, Wain G. Breast cancer survivors' supportive care needs 2-10 years after diagnosis. Support Care Cancer. 2007; 15: 515-523. doi:10.1007/s00520-006-0170-2

16. He $Y$, Guo X, May BH, et al. Clinical evidence for association of acupuncture and acupressure with improved cancer pain. JAMA Oncol. 2020; 6: 271-278. doi:10.1001/jamaoncol.2019.5233

17. Pfister DG, Cassileth BR, Deng GE, et al. acupuncture for pain and dysfunction after neck dissection: Results of a randomized controlled trial. J Clin Oncol. 2010; 28: 2565-2570. doi:10.1200/JCO.2009.26.9860

18. Hammar M, Frisk J, Grimås $O$, Höök $M$, Spetz AC, Wyon Y. Acupuncture treatment of vasomotor symptoms in men with prostatic carcinoma: A pilot study. J Urol. 1999; 161: 853856. http://www.ncbi.nlm.nih.gov/pubmed/10022700.

19. Pfister DG, Cassileth BR, Deng GE, et al. Acupuncture for pain and dysfunction after neck dissection: results of a randomized controlled trial. J Clin Oncol. 2010; 28: 2565-2570. doi:10.1200/JCO.2009.26.9860

20. Ezzo J, Vickers A, Richardson MA, et al. Acupuncture-point stimulation for chemotherapyinduced nausea and vomiting. J Clin Oncol. 2005; 23: 7188-7198. doi:10.1200/JCO.2005.06.028

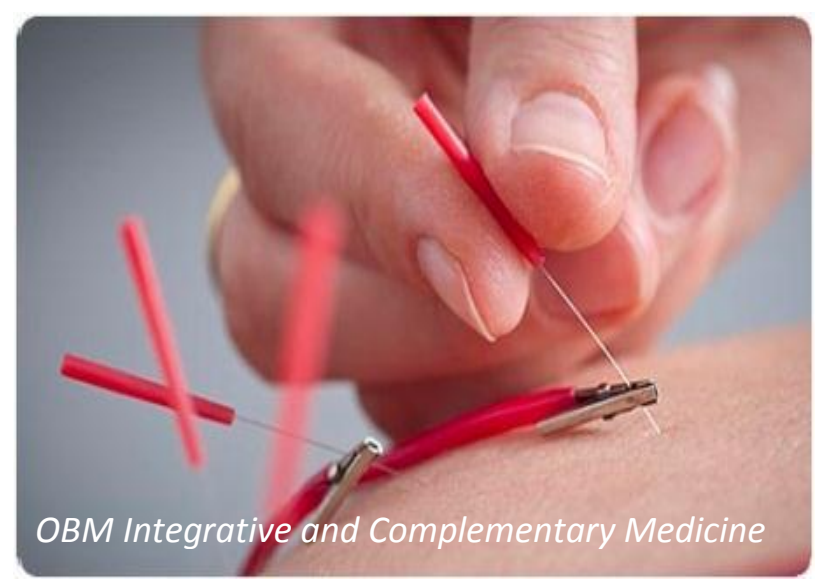

Enjoy OBM Integrative and Complementary Medicine by:

1. Submitting a manuscript

2. Joining in volunteer reviewer bank

3. Joining Editorial Board

4. Guest editing a special issue

For more details, please visit:

http://www.lidsen.com/journals/icm 